\title{
In Vitro and In Vivo Interaction Studies Between Lesinurad, a Selective Urate Reabsorption Inhibitor, and Major Liver or Kidney Transporters
}

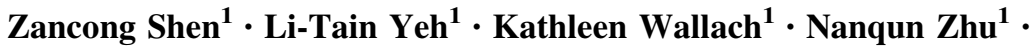 \\ Brad Kerr ${ }^{1} \cdot$ Michael Gillen $^{2}$
}

Published online: 7 March 2016

(c) The Author(s) 2016. This article is published with open access at Springerlink.com

\begin{abstract}
Background and Objectives Lesinurad is a selective uric acid reabsorption inhibitor (SURI) under investigation for the treatment of gout. This study elucidated the interaction of lesinurad with major liver and kidney transporters in vitro and evaluated the drug-drug interactions (DDIs) of lesinurad and atorvastatin, metformin, and furosemide in clinical studies.

Methods Lesinurad interaction with membrane transporters was evaluated in validated transporter-expressing cell systems and analyzed by liquid scintillation counting. Healthy male subjects (ages 18-65 years; body mass index $\left.18-32 \mathrm{~kg} / \mathrm{m}^{2}\right)$ received atorvastatin $(40 \mathrm{mg} ; n=28)$ with or without lesinurad 200 or $400 \mathrm{mg}$, or received metformin ( $850 \mathrm{mg} ; n=12$ ) or furosemide ( $40 \mathrm{mg} ; n=11$ ) with or without lesinurad $400 \mathrm{mg}$. Plasma concentrations of each concomitant drug were determined by validated liquid chromatography with tandem mass spectrometry methods. Results Lesinurad interacted in vitro with OATP1B1, OCT1, and OAT1/3 transporters. Co-administration of lesinurad $200 \mathrm{mg}$ did not significantly alter plasma exposure (maximum concentration $\left[C_{\max }\right]$ and area under the concentration-time curve [AUC]) of total atorvastatin (atorvastatin + hydroxyl-metabolites) or atorvastatin, while co-administration of lesinurad $400 \mathrm{mg}$ increased the $C_{\max }$ of total atorvastatin and atorvastatin by $17-26 \%$, but had no effect on AUC. Co-administration of lesinurad
\end{abstract}

Zancong Shen

ZShen@ardeabio.com

1 Translational Sciences, Ardea Biosciences, Inc., 9390 Towne Centre Drive, San Diego, CA 92121, USA

2 AstraZeneca LP, One MedImmune Way, Gaithersburg, MD 20878, USA
$400 \mathrm{mg}$ had no effect on the plasma exposure of metformin. Furosemide plasma AUC was reduced by $31 \%$ in the presence of lesinurad $400 \mathrm{mg}$, but furosemide renal clearance and diuretic activity were unchanged.

Conclusions No clinically relevant DDIs were observed between lesinurad and substrates of major liver or kidney transporters.

\section{Key Points}

Potential interactions between lesinurad and major liver or kidney transporters were identified in vitro.

Atorvastatin, metformin, and furosemide are drugs that interact with OATP1B1/3, OCT1/2, and OAT1/3 transporters.

Drug-drug interaction studies showed no clinically relevant interaction between lesinurad and these drugs.

\section{Introduction}

Gout is a chronic progressive inflammatory arthritis characterized by the deposition of monosodium urate crystals in musculoskeletal structures (e.g., joints), kidneys, and other connective tissues, which results from high concentrations of serum uric acid (sUA) [1]. For long-term management of gout, maintenance of sUA levels below $6.0 \mathrm{mg} / \mathrm{dL}$ (below $5.0 \mathrm{mg} / \mathrm{dL}$ for greater disease severity) with urate-lowering therapy is recommended [2]. The recommended first line of therapy are the xanthine oxidase inhibitors (XOIs) 
allopurinol and febuxostat that reduce uric acid production to lower sUA [2-4]. If the target sUA level cannot be maintained with an XOI at the appropriate dose, treatment guidelines recommend combination therapy that includes an XOI with a uricosuric [2-4].

Lesinurad (Zurampic ${ }^{\circledR}$; AstraZeneca Pharmaceuticals, Wilmington, DE, USA) is a novel selective uric acid reabsorption inhibitor (SURI) approved in the USA for the treatment of gout in combination with an XOI. Lesinurad inhibits URAT1, the uric acid transporter mainly responsible for reabsorption of uric acid from the glomerular ultrafiltrate into the epithelial cells of the renal proximal convoluted tubule [5]. The combination of lesinurad and an XOI lowers sUA by increasing fractional excretion of uric acid [6] and reducing uric acid production.

Drug exposure is dependent on the absorption, distribution, metabolism, and excretion of the drug, and transporters expressed in the kidney and liver have been increasingly acknowledged to play a major role in these processes [7]. These transporters are classified into two superfamilies: the solute carrier family (including organic anion transporter [OAT], organic anion transporter polypeptide [OATP], organic cation transporter [OCT], and multidrug and toxic exclusion [MATE] transporters) and the ATP-binding cassette protein family (such as permeability glycoprotein [Pgp] and breast cancer resistance protein [BCRP]) [7, 8]. The US Food and Drug Administration (FDA) has recommended conducting in vitro and in vivo studies on a specific group of transporters, including OATP1B1, OATP1B3, OAT1, OAT3, and OCT2, during drug development to evaluate the potential for drug-drug interactions [9].

Here, we report the in vitro interaction of lesinurad with major liver and kidney transporters. We also report clinical studies undertaken to evaluate the potential inhibitory effect of single doses of lesinurad (200 and $400 \mathrm{mg}$ ) on the pharmacokinetics of a single dose of atorvastatin, metformin, or furosemide, all of which are known substrates for major liver or kidney transporters [7].

\section{Methods}

\subsection{In Vitro Studies}

Validated oocytes, HEK293, MDCK-II, Caco-2 or MDCKMDR1 cell systems were used to study the interaction of lesinurad with membrane transporters localized to the kidney (OAT1, OAT3, OCT2, MATE1, and MATE2K) or liver (Pgp, BCRP, OATP1B1, OATP1B3, and OCT1) [10, 11]. Xenopus laevis oocytes were injected with OAT1 or OAT3 cRNA or control (water) while HEK293 cells were stably transfected with MATE1, MATE2K, or vector and MDCK-II cells with hOATP1B1, hOATP1B3, hOCT1, hOCT2, or vector. The MDCKII cell line was stably transfected with the human MDR1 gene to create a P-gp cell line. The interaction of lesinurad with BCRP relied on the endogenous expression in Caco- 2 cells. All cells were cultured with growth medium according to standard methodology. In order to determine whether lesinurad was a substrate for a transporter, cells were incubated with $\left[{ }^{14} \mathrm{C}\right]$-labeled lesinurad at various concentrations and the amount of lesinurad taken up by the cells determined by subtracting the uptake in vector cells from that in the transfected cells. The uptake of a $\left[{ }^{3} \mathrm{H}\right]$-labeled known substrate of the transporter served as the positive control. Inhibition of a transporter by lesinurad was determined by incubating cells with a fixed concentration of $\left[{ }^{3} \mathrm{H}\right]$-labeled known substrate and various concentrations of unlabeled lesinurad. Inhibition by a known inhibitor of each transporter served as the positive control. Cells were incubated for the appropriate amount of time (see Table 1). All reactions were terminated by the addition of ice-cold medium. The cells were then rinsed with medium and lysed.

\subsubsection{Analytical Method}

Uptake of $\left[{ }^{14} \mathrm{C}\right]$-labeled lesinurad or $\left[{ }^{3} \mathrm{H}\right]$-labeled known substrates was measured by liquid scintillation counting (Perkin Elmer, Waltham, MA, USA and Hitachi Aloka, Tokyo, Japan). Transporter-mediated uptake was calculated by subtracting uptake in the non-transfected cells from uptake in the transfected cells. The inhibitor concentration giving half-maximum inhibition $\left(\mathrm{IC}_{50}\right)$ was calculated by:

$\%$ of control $=100 \times \mathrm{IC}_{50} /\left(\mathrm{IC}_{50}+[I]\right)$

where $[I]$ is the inhibitor concentration $(\mu \mathrm{M})$.

The $\mathrm{IC}_{50}$ was not calculated if inhibition $\geq 50 \%$ was not observed at any tested lesinurad concentration.

\subsection{Clinical Pharmacology Studies}

\subsubsection{Study Design}

The two clinical studies were open-label, randomized, phase I studies to investigate the effect of single-dose lesinurad on the pharmacokinetics of atorvastatin, metformin, or furosemide in healthy male subjects.

In the first study, a two-cohort design was adopted for evaluation of single doses of lesinurad with atorvastatin, in which subjects were randomized $1: 1$ to either lesinurad $200 \mathrm{mg}$ with or without atorvastatin or lesinurad $400 \mathrm{mg}$ with or without atorvastatin. Subjects received lesinurad 200 or $400 \mathrm{mg}$ on day 4 and a single dose of atorvastatin $40 \mathrm{mg}$ on days 1 and 4. Atorvastatin and lesinurad were given in the morning, approximately $30 \mathrm{~min}$ after consumption of a standard breakfast. The study was conducted 
Table 1 In vitro inhibition of kidney and liver transporters by lesinurad and known inhibitors of each transporter

\begin{tabular}{|c|c|c|c|c|c|c|c|}
\hline Transporter & $\begin{array}{l}\text { Cell type/incubation } \\
\text { time }\end{array}$ & Substrate & Inhibitor & $\begin{array}{l}\% \\
\text { inhibition }\end{array}$ & $\begin{array}{l}\text { Lesinurad } \\
\mathrm{IC}_{50}, \mu \mathrm{M}\end{array}$ & $\begin{array}{l}\text { Lesinurad as } \\
\text { substrate }\end{array}$ & $\begin{array}{l}C_{\max } / \\
\mathrm{IC}_{50}{ }^{\mathrm{a}}\end{array}$ \\
\hline \multicolumn{8}{|c|}{ Kidney transporters } \\
\hline \multirow[t]{3}{*}{ OAT1 } & \multirow[t]{3}{*}{ Oocytes/60 min } & \multirow[t]{3}{*}{$\begin{array}{l}3 \mu \mathrm{M} \text { para- } \\
\text { aminohippurate }\end{array}$} & $\begin{array}{l}300 \mu \mathrm{M} \\
\text { probenecid }\end{array}$ & 99 & \multirow[t]{3}{*}{4.3} & \multirow[t]{3}{*}{ Yes } & \multirow[t]{3}{*}{0.06} \\
\hline & & & $5 \mu \mathrm{M}$ lesinurad & 46 & & & \\
\hline & & & $25 \mu \mathrm{M}$ lesinurad & 87 & & & \\
\hline \multirow[t]{3}{*}{ OAT3 } & \multirow[t]{3}{*}{ Oocytes/60 min } & \multirow[t]{3}{*}{$2 \mu \mathrm{M}$ estrone-3-sulfate } & $\begin{array}{l}200 \mu \mathrm{M} \\
\text { bumetanide }\end{array}$ & 97 & \multirow[t]{3}{*}{3.5} & \multirow[t]{3}{*}{ Yes } & \multirow[t]{3}{*}{0.08} \\
\hline & & & $5 \mu \mathrm{M}$ lesinurad & 64 & & & \\
\hline & & & $25 \mu \mathrm{M}$ lesinurad & 95 & & & \\
\hline \multirow[t]{5}{*}{ OCT2 } & \multirow[t]{5}{*}{ MCDK-II/5 min } & \multirow[t]{5}{*}{$10 \mu \mathrm{M}$ metformin } & $100 \mu \mathrm{M}$ quinidine & 78 & \multirow[t]{5}{*}{$>300$} & \multirow[t]{5}{*}{ No } & \multirow[t]{5}{*}{$<0.001$} \\
\hline & & & $3 \mu \mathrm{M}$ lesinurad & 10 & & & \\
\hline & & & $30 \mu \mathrm{M}$ lesinurad & 6 & & & \\
\hline & & & $100 \mu \mathrm{M}$ lesinurad & 26 & & & \\
\hline & & & $300 \mu \mathrm{M}$ lesinurad & 18 & & & \\
\hline \multirow[t]{4}{*}{ MATE1 } & \multirow[t]{4}{*}{ HEK293/2 min } & \multirow[t]{4}{*}{$\begin{array}{l}5 \mu \mathrm{M} \\
\text { tetraethylammonium }\end{array}$} & $10 \mu \mathrm{M}$ cimetidine & 88 & \multirow[t]{4}{*}{$\begin{array}{l}\text { Not } \\
\text { calculated }\end{array}$} & \multirow[t]{4}{*}{$\begin{array}{l}\text { Not } \\
\text { evaluated }\end{array}$} & \multirow[t]{4}{*}{ NA } \\
\hline & & & $1 \mu \mathrm{M}$ lesinurad & 9 & & & \\
\hline & & & $10 \mu \mathrm{M}$ lesinurad & 2 & & & \\
\hline & & & $100 \mu \mathrm{M}$ lesinurad & 20 & & & \\
\hline \multirow[t]{4}{*}{ MATE2K } & HEK293/2 min & $\begin{array}{l}10 \mu \mathrm{M} \\
\text { tetraethylammonium }\end{array}$ & $100 \mu \mathrm{M}$ cimetidine & 76 & $\begin{array}{l}\text { Not } \\
\text { calculated }\end{array}$ & $\begin{array}{l}\text { Not } \\
\text { evaluated }\end{array}$ & NA \\
\hline & & & $1 \mu \mathrm{M}$ lesinurad & -21 & & & \\
\hline & & & $10 \mu \mathrm{M}$ lesinurad & -16 & & & \\
\hline & & & $100 \mu \mathrm{M}$ lesinurad & -9 & & & \\
\hline Liver transpo & rters & & & & & & \\
\hline OATP1B1 & MCDK-II/5 min & $2 \mu \mathrm{M}$ & $100 \mu \mathrm{M}$ rifampicin & 99 & 9.3 & Yes & 1.8 \\
\hline & & bromosulfophthalein & $3 \mu \mathrm{M}$ lesinurad & 25 & & & \\
\hline & & & $30 \mu \mathrm{M}$ lesinurad & 80 & & & \\
\hline OATP1B3 & MCDK-II/5 min & $2 \mu \mathrm{M}$ & $100 \mu \mathrm{M}$ rifampicin & 98 & 43.1 & Minor & 0.39 \\
\hline & & bromosulfophthalein & $3 \mu \mathrm{M}$ lesinurad & 21 & & & \\
\hline & & & $30 \mu \mathrm{M}$ lesinurad & 49 & & & \\
\hline OCT1 & MCDK-II/5 min & $10 \mu \mathrm{M}$ metformin & $100 \mu \mathrm{M}$ quinidine & 77 & 13.7 & Yes & 2.9 \\
\hline & & & $3 \mu \mathrm{M}$ lesinurad & 36 & & & \\
\hline & & & $30 \mu \mathrm{M}$ lesinurad & 58 & & & \\
\hline BCRP & Caco-2/120 min & $25 \mathrm{nM}$ genistein & $100 \mu \mathrm{M}$ chrysin & 92 & $>3000$ & Minor & $<0.006$ \\
\hline & & & $3 \mu \mathrm{M}$ lesinurad & 18 & & & \\
\hline & & & $30 \mu \mathrm{M}$ lesinurad & 27 & & & \\
\hline & & & $100 \mu \mathrm{M}$ lesinurad & 38 & & & \\
\hline P-gp & $\begin{array}{l}\text { MDCK-MDR1/ } \\
120 \mathrm{~min}\end{array}$ & $100 \mathrm{nM}$ digoxin & $\begin{array}{l}50 \mu \mathrm{M} \\
\text { ketoconazole }\end{array}$ & 100 & 1000 & No & 0.02 \\
\hline & & & $3 \mu \mathrm{M}$ lesinurad & -6 & & & \\
\hline & & & $30 \mu \mathrm{M}$ lesinurad & -3 & & & \\
\hline & & & $100 \mu \mathrm{M}$ lesinurad & -6 & & & \\
\hline
\end{tabular}

$B C R P$ breast cancer resistance protein, $C_{\max }$ maximum concentration, $I C_{50}$ half maximum inhibitory concentration, $O A T$ organic anion transporter, $O A T P$ organic anion transporter polypeptide, $O C T$ organic cation transporter, MATE multidrug and toxic exclusion, $P$-gP permeability glycoprotein

${ }^{\mathrm{a}} C_{\max } / \mathrm{IC}_{50}$ ratio: for kidney transporter, $C_{\max }(200 \mathrm{mg}$ dose $)$ is approximately $17.1 \mu \mathrm{M}$, using $98.4 \%$ as protein-bound fraction for the calculation of free lesinurad concentration; for liver transporter, $C_{\max }(200 \mathrm{mg}$ dose) total concentration (approximately $17.1 \mu \mathrm{M})$ was used 
at a single clinical research facility (Overland Park, KS, USA) between 11 June 2011 and 1 August 2011.

In the second study, a two-sequence, two-period, crossover design was used to evaluate single-dose lesinurad $400 \mathrm{mg}$ in combination with metformin $850 \mathrm{mg}$ or furosemide $40 \mathrm{mg}$ in subjects randomized $1: 1$ to one of two sequences within each separate cohort: metformin or furosemide on day 1 followed by lesinurad plus metformin or furosemide on day 5 or lesinurad plus metformin or furosemide on day 1 followed by metformin or furosemide on day 5. Lesinurad and metformin or lesinurad and furosemide were orally administered with $240 \mathrm{~mL}$ of water after an overnight fast of at least $10 \mathrm{~h}$. No food was allowed for $4 \mathrm{~h}$ after the study treatments were given. Subjects were required to remain well hydrated, with a minimum fluid intake of 2-2.5 L per day. A larger fluid intake was necessary for furosemide-treated subjects, in whom consumption was guided by thirst. The study was conducted at a single clinical research facility (Kalamazoo, MI, USA) between 29 October 2013 and 18 December 2013.

The studies were conducted in accordance with the Declaration of Helsinki and International Conference on Harmonisation Good Clinical Practice (ICH GCP) guidelines. The protocol, protocol amendments, and written informed consent form were approved by an institutional review board. Subjects provided written informed consent prior to study participation.

\subsubsection{Study Population}

Males between 18 and 65 years of age, with a body weight above $50 \mathrm{~kg}$ and a body mass index within the range of 18 and $30 \mathrm{~kg} / \mathrm{m}^{2}$, were eligible. Subjects were also required to be free of significant disease necessitating physician care or interfering with study evaluations or procedures and to have normal measurements for laboratory parameters (chemistry, hematology, urinalysis), physical examination, vital signs, or electrocardiogram.

Key exclusion criteria were inadequate venous access or unsuitable veins for repeated venipuncture; concomitant chronic or acute illness or an acute febrile illness within 1 week of dose administration; history or current symptoms of renal calculi or gout; major surgery within 3 months prior to first dose; clinically relevant intolerance or allergy to foods or drugs (including statins), or known or suspected hypersensitivity to any ingredient in the investigational products; history of drug addiction or excessive alcohol use; and previous participation in a lesinurad study.

\subsubsection{Pharmacokinetic Sample Collection}

For determination of atorvastatin pharmacokinetics, blood samples were collected on day 1 and day 4 pre-dose (within
30 min before dosing) and at $0.25,0.5,1,1.5,2,3,4,5,6$, $8,12,16,24,30,36,48,60$, and $72 \mathrm{~h}$ post-dose. For metformin or furosemide, blood samples were collected at the following times on days 1 and 5: pre-dose (within 30 min before dosing) and at 0.5, 1, 1.5, 2, 2.5, 3, 4, 5, 6, 8, $10,12,14$, and $24 \mathrm{~h}$ post-dose. Urine pharmacokinetics were assessed over post-dose intervals of $0-6,6-12$, and 12-24 h on day 1 and day 5 of treatment with furosemide.

\subsubsection{Pharmacokinetic Sample Analyses}

All analyses were conducted using previously validated highperformance liquid chromatography-tandem mass spectrometry (HPLC-MS/MS) methods. Plasma samples were analyzed for atorvastatin and metabolites by Tandem Labs (Salt Lake City, UT, USA) and for metformin and furosemide by PPD (Richmond, VA, USA). Urine samples were analyzed for furosemide by inVentive Health Clinique, Inc. (Quebec City, Quebec, Canada). In plasma, the calibration range was $0.3-50 \mathrm{ng} / \mathrm{mL}$ for atorvastatin, $2-\mathrm{OH}$ atorvastatin and $4-\mathrm{OH}$ atorvastatin; $2.00-1000 \mathrm{ng} / \mathrm{mL}$ for metformin; and $5.00-5000 \mathrm{ng} / \mathrm{mL}$ for furosemide. In urine, the calibration range was $100-200,000 \mathrm{ng} / \mathrm{mL}$ for furosemide.

\subsubsection{Statistical Analysis: Pharmacokinetic and Pharmacodynamic Analyses}

Plasma pharmacokinetic parameters were derived using validated WinNonlin platform (Pharsight Corporation, Mountain View, CA, USA). The maximal plasma concentration $\left(C_{\max }\right)$ and the time to reach $C_{\max }\left(T_{\max }\right)$ were calculated from individual plasma concentration-time profiles of atorvastatin (including the metabolites 2-OHatorvastatin and 4-OH-atorvastatin), metformin or plasma and urine profiles of furosemide using a non-compartmental method and summarized by treatment. Additional calculated pharmacokinetic parameters included the area under the concentration-time curve (AUC) from time zero to the time of the last quantifiable concentration $\left(\mathrm{AUC}_{\text {last }}\right)$, AUC from time zero to infinity $\left(\mathrm{AUC}_{\infty}\right)$, and apparent terminal half-life. Additional pharmacokinetic parameters for metformin and furosemide included apparent total body clearance and volume of distribution at steady state.

Geometric mean (GMR) point estimates and $90 \%$ confidence intervals (CIs) of treatment differences were calculated. For the atorvastatin study, no DDI was deemed to have occurred if the GMR was contained within the boundaries of equivalence (80-125\%). For the metformin and furosemide studies, the usual criterion was employed where no DDI was deemed to have occurred if the $90 \%$ CIs of the GMR were contained within the 80-125\% boundaries. For uniformity, the results with atorvastatin were also analyzed using the latter criterion. 
An ad hoc pharmacodynamic analysis was conducted to assess for change in the diuretic properties of furosemide, including urine sodium, urine volume, and specific gravity in the presence of lesinurad. These analyses were performed using a mixed model that incorporated treatment, time and treatment by time interaction as fixed effects. Least squares means and $P$ values were calculated for the comparison between lesinurad plus furosemide and furosemide alone.

\section{Results}

\subsection{In Vitro Analyses}

Lesinurad was determined to be a substrate for the kidney transporters OAT1 and OAT3 with $K_{\mathrm{m}}$ values of 0.85 and $2 \mu \mathrm{M}$, respectively; lesinurad was not a substrate for OCT2 and was not evaluated for MATE1 or MATE2K (Table 1). Of the liver transporters, lesinurad was a weak substrate for OCT1 $\left(K_{\mathrm{m}}\right.$ of $\left.\sim 20 \mu \mathrm{M}\right)$ and OATP1B1. As a substrate for OATP1B1, lesinurad was only tested at $10 \mu \mathrm{M}$, where a 2.25-fold increase in lesinurad uptake was observed in transfected cells versus non-transfected cells. Lesinurad was a minor substrate for BCRP, but was not a substrate for OATP1B3 or P-gp.

Inhibition of the transport of known substrates of major kidney and liver transporters by lesinurad and known inhibitors is shown in Table 1 . The greatest inhibition by lesinurad was observed with the kidney transporters OAT1 and OAT3 and the liver transporter OATP1B1, less inhibition was observed with OCT1 and OATP1B3, and little or no inhibition with OCT2, P-gp, BCRP, MATE1, and MATE2K. The calculated $C_{\max } / \mathrm{IC}_{50}$ ratio suggested that lesinurad may have a higher potential to inhibit OATP1B1 (1.8) followed by OCT1 (1.2) and OATP1B3 (0.39) clinically and is not considered to be an inhibitor of OAT1 (0.06), OAT3 (0.08), P-gp (0.02), BCRP $(<0.006)$, OCT 2 $(<0.001)$, MATE1, or MATE2K. To assess the in vivo relevance of these in vitro findings, clinical pharmacology studies evaluated the effect of lesinurad on the pharmacokinetics of atorvastatin (OATP1B1 substrate), metformin (OCT1 substrate), and furosemide (OAT1/3 substrate).

\subsection{Clinical Pharmacology Studies}

\subsubsection{Study Population}

In the first study, 28 subjects were randomized to lesinurad $200 \mathrm{mg}(n=14)$ or $400 \mathrm{mg}(n=14)$ and atorvastatin. All 28 completed the study. The study population comprised male subjects, white (46.4\%) and black (46.4\%), with a mean (SD) age of 35 (13) years and body mass index of $26.6(2.4) \mathrm{kg} / \mathrm{m}^{2}$.
In the second study, 23 subjects received lesinurad and metformin $(n=12)$ or furosemide $(n=11)$; none discontinued the study. Subjects were male, white $(91.3 \%)$, with a mean (SD) age of 33 (9) years and body mass index of $24.9(2.5) \mathrm{kg} / \mathrm{m}^{2}$. Baseline characteristics were similar between groups in both studies.

\subsubsection{Effect of Lesinurad on Atorvastatin Pharmacokinetics}

The plasma concentration-time profile of a single dose of atorvastatin $40 \mathrm{mg}$ alone and in combination with a single dose of lesinurad $200 \mathrm{mg}$ or in combination with lesinurad $400 \mathrm{mg}$ are presented in Fig. 1a, b, respectively. Atorvastatin exposure, as noted by the geometric mean $(95 \% \mathrm{CI})$ for $C_{\max }$ and AUC, was not significantly altered by coadministration with lesinurad at either dose (Table 2). No DDI between atorvastatin and $200 \mathrm{mg}$ lesinurad was deemed to have occurred as the GMR and the $90 \% \mathrm{CI}$ of the GMR for $C_{\max }$ and $\mathrm{AUC}_{\infty}$ were contained within the 80-125\% boundaries (Table 3). Lesinurad did increase the $C_{\text {max }}(16 \%)$ and $\mathrm{AUC}_{\infty}(20 \%)$ of $2-\mathrm{OH}$-atorvastatin. The $C_{\max }$ of atorvastatin, total atorvastatin and 2-OH-atorvastatin was increased $17-34 \%$ by the addition of lesinurad $400 \mathrm{mg}$, as the upper bound of the $90 \%$ CIs were above the $125 \%$ upper boundary. The AUC for atorvastatin, total atorvastatin, and 2-OH-atorvastatin were not significantly altered by the co-administration of lesinurad (Table 3 ). The minor metabolite, 4-OH-atorvastatin, was evident at only trace plasma concentration levels, so it was not possible to characterize its pharmacokinetics.

\subsubsection{Effect of Lesinurad on Metformin or Furosemide Pharmacokinetics}

The plasma concentration-time profile of a single dose of metformin $850 \mathrm{mg}$ alone and in combination with a single dose of lesinurad $400 \mathrm{mg}$, and a single dose of furosemide $40 \mathrm{mg}$ alone and in combination with lesinurad $400 \mathrm{mg}$ are presented in Fig. 1c, d, respectively. With metformin, there were no marked differences in the GMR (95\% CI) for metformin pharmacokinetic parameters in the presence versus absence of lesinurad (Table 2). The $90 \%$ CIs around the GMRs for metformin $C_{\max }$ and AUC were entirely contained within the 80-125\% boundaries (Table 3).

With furosemide, the co-administration of lesinurad increased median $T_{\max }$ from $1 \mathrm{~h}$ to $2 \mathrm{~h}$ post-dose, and $C_{\max }$ and AUC values were lower than with furosemide alone (Table 2). Co-administration with lesinurad reduced the furosemide $C_{\max }$ by $51 \%$ and $\mathrm{AUC}$ by $31 \%$, with the $90 \%$ CIs around the GMR for both $C_{\max }$ and AUC not contained within the $80-125 \%$ boundaries (Table 3 ). 
(a)

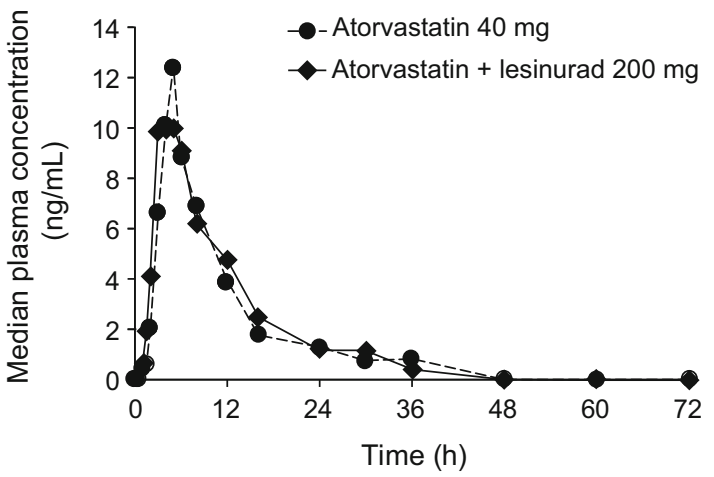

(c)

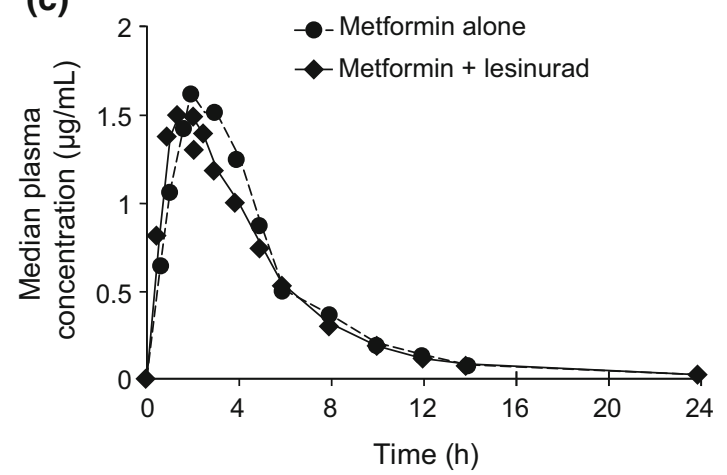

Fig. 1 Median plasma concentration profiles for total atorvastatin (including metabolites) following a single oral dose of atorvastatin $40 \mathrm{mg}$ in the absence or presence of a single dose of lesinurad $200 \mathrm{mg}$ (a) or $400 \mathrm{mg}$ (b), and plasma concentration profile of metformin

Consistent with the reduction in plasma exposure, the amount of furosemide excreted in urine was reduced by approximately $25 \%$ in the presence of lesinurad. The GMR $(95 \% \mathrm{CI})$ for renal clearance $\left(\mathrm{CL}_{\mathrm{R}}\right)$ of furosemide was $99.3(89.2-111) \mathrm{mL} / \mathrm{min}$ and $107(97.5-119) \mathrm{mL} / \mathrm{min}$ in the absence and presence of lesinurad, respectively. $\mathrm{CL}_{\mathrm{R}}$ was unchanged by lesinurad, as the GMR and the $90 \% \mathrm{CI}$ around the GMR for $\mathrm{CL}_{\mathrm{R}}$ fell within the 80-125\% boundaries (Table 3 ).

There was no clinically significant impact of lesinurad on the activity of furosemide based on an assessment of urine flow rate, volume, sodium concentration, and specific gravity at each urine collection interval (Table 4).

\section{Discussion}

There is increasing awareness of the importance of understanding DDIs between gout treatments and concomitantly administered drugs [12, 13]. A series of in vitro studies were undertaken to establish the potential for transporter-mediated DDIs between lesinurad and commonly used drugs in patients with gout following the (b)

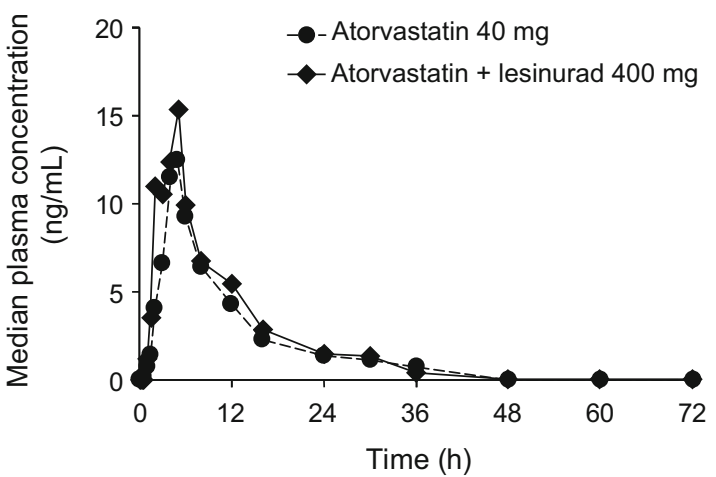

(d)

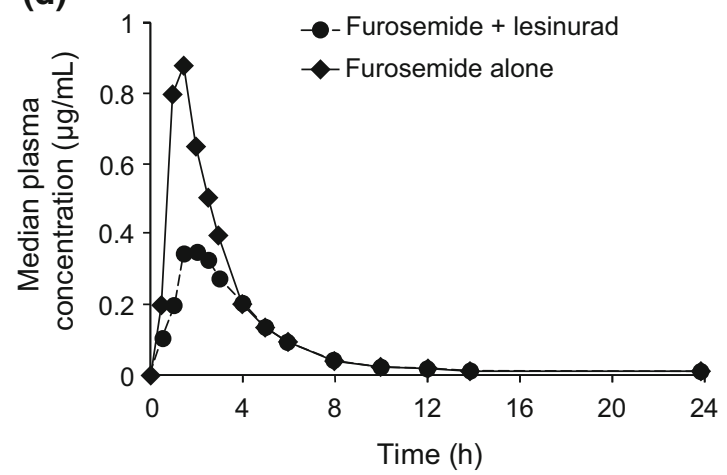

following a single dose of metformin $850 \mathrm{mg}$ (c) or plasma concentration profile of furosemide following a single dose of furosemide $40 \mathrm{mg}$ (d) in the absence or presence of a single dose of lesinurad $400 \mathrm{mg}$

FDA Drug Interaction Guidance [9]. Using validated in vitro cell systems expressing specific transport proteins, it was shown that lesinurad was associated with a potential to inhibit the liver transporter OATP1B1 and, to a lesser extent, OCT1 and OATP1B3. The in vitro investigations also indicated that inhibition of the major kidney transporters, OAT1 and OAT3, by lesinurad was minimal, and no inhibition of OCT2 was expected. Results from the in vitro analyses also suggested that lesinurad is unlikely to exert an effect on MATE1 and MATE2K, which are transporters involved in the regulation of serum creatinine and the renal elimination of drugs $[14,15]$.

DDIs between lesinurad and commonly used drugs known to be substrates of the kidney or liver transporters identified in the in vitro analyses were investigated in clinical pharmacology studies. Atorvastatin is a substrate of the liver transporter OATP1B1 [7, 16, 17], which was identified as potentially being inhibited by lesinurad. However, our study showed that lesinurad $200 \mathrm{mg}$ did not significantly alter the pharmacokinetics of atorvastatin, while there was a slight increase in atorvastatin exposure with lesinurad $400 \mathrm{mg}$. The marginal changes in 


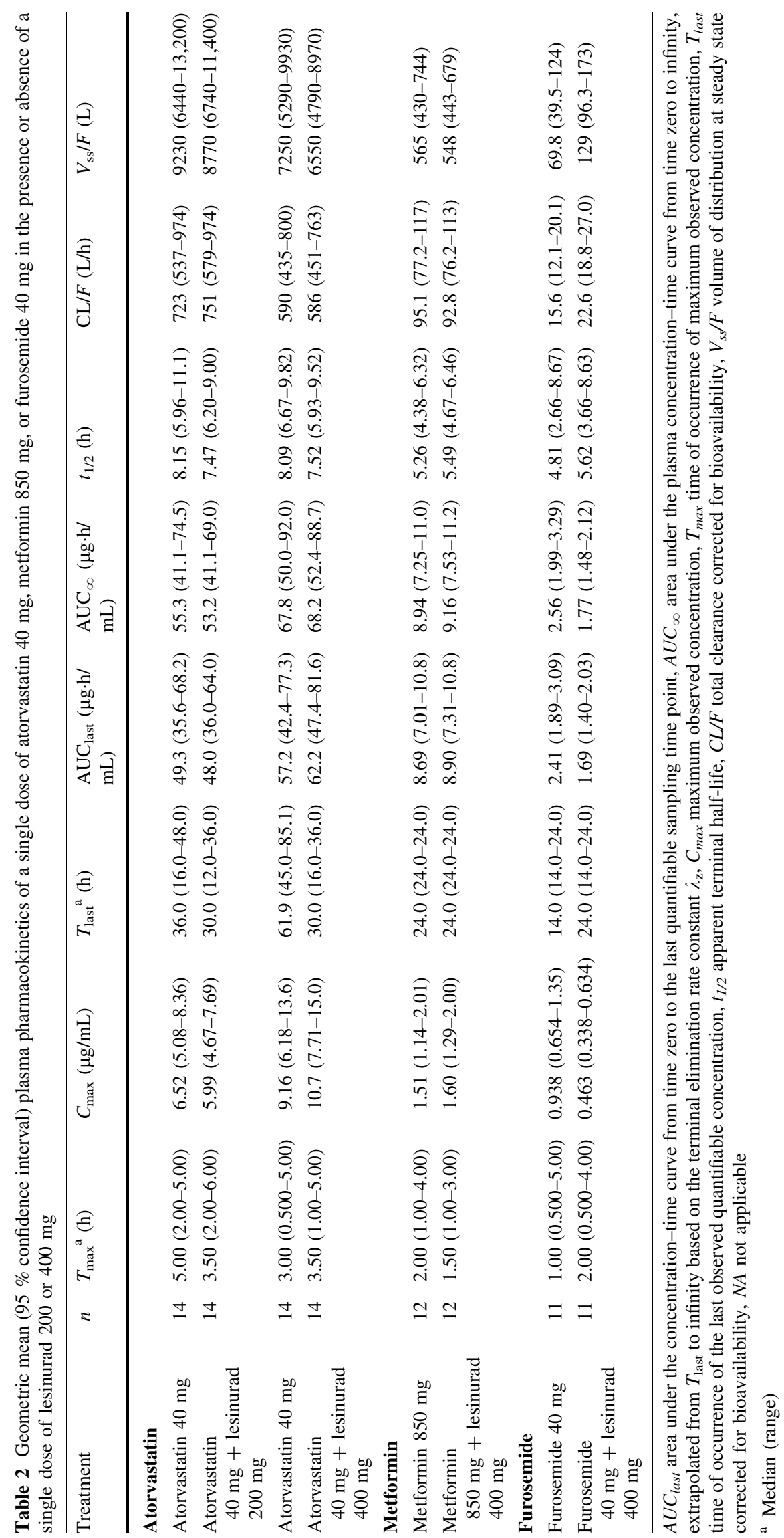


Table 3 Geometric mean ratios (GMRs) (90\% confidence interval) for atorvastatin, metformin, and furosemide in the presence versus absence of lesinurad

\begin{tabular}{|c|c|c|c|}
\hline Treatment & Analyte & Parameter & GMR $(90 \% \mathrm{CI})$ \\
\hline \multirow[t]{6}{*}{ Atorvastatin $40 \mathrm{mg}+$ lesinurad $200 \mathrm{mg}$ vs. atorvastatin $40 \mathrm{mg}(n=14)$} & \multirow[t]{2}{*}{ Atorvastatin } & $C_{\max }$ & $0.919(0.803-1.05)$ \\
\hline & & $\mathrm{AUC}_{\infty}$ & $0.962(0.898-1.03)$ \\
\hline & \multirow[t]{2}{*}{ Total atorvastatin } & $C_{\max }$ & $1.01(0.879-1.16)$ \\
\hline & & $\mathrm{AUC}_{\infty}$ & $1.07(0.980-1.17)$ \\
\hline & \multirow[t]{2}{*}{ 2-OH-atorvastatin } & $C_{\max }$ & $1.16(0.991-1.36)$ \\
\hline & & $\mathrm{AUC}_{\infty}$ & $1.20(1.08-1.34)$ \\
\hline \multirow[t]{6}{*}{ Atorvastatin $40 \mathrm{mg}+$ lesinurad $400 \mathrm{mg}$ vs. atorvastatin $40 \mathrm{mg}(n=14)$} & \multirow[t]{2}{*}{ Atorvastatin } & $C_{\max }$ & $1.17(0.940-1.46)$ \\
\hline & & $\mathrm{AUC}_{\infty}$ & $1.01(0.913-1.11)$ \\
\hline & \multirow[t]{2}{*}{ Total atorvastatin } & $C_{\max }$ & $1.26(1.05-1.50)$ \\
\hline & & $\mathrm{AUC}_{\infty}$ & $1.08(1.00-1.18)$ \\
\hline & \multirow[t]{2}{*}{ 2-OH-atorvastatin } & $C_{\max }$ & $1.34(1.15-1.55)$ \\
\hline & & $\mathrm{AUC}_{\infty}$ & $1.17(1.07-1.27)$ \\
\hline \multirow[t]{3}{*}{ Metformin $850 \mathrm{mg}+$ lesinurad $400 \mathrm{mg}$ vs. metformin $850 \mathrm{mg}(n=12)$} & \multirow[t]{3}{*}{ Metformin } & $C_{\max }$ & $1.06(1.00-1.13)$ \\
\hline & & $\mathrm{AUC}_{\text {last }}$ & $1.02(0.908-1.15)$ \\
\hline & & $\mathrm{AUC}_{\infty}$ & $1.03(0.911-1.15)$ \\
\hline \multirow[t]{5}{*}{ Furosemide $40 \mathrm{mg}$ + lesinurad $400 \mathrm{mg}$ vs. furosemide $40 \mathrm{mg}(n=11)$} & \multirow[t]{5}{*}{ Furosemide } & $C_{\max }$ & $0.489(0.387-0.618)$ \\
\hline & & $\mathrm{AUC}_{\text {last }}$ & $0.697(0.588-0.826)$ \\
\hline & & $\mathrm{AUC}_{\infty}$ & $0.693(0.567-0.847)$ \\
\hline & & $\mathrm{Ae}_{0-24}$ & $0.752(0.649-0.872)$ \\
\hline & & $\mathrm{CL}_{\mathrm{R}(0-24)}$ & $108(101-116)$ \\
\hline
\end{tabular}

$C_{\max }$ maximum observed concentration, $A U C_{\text {last }}$ area under the concentration-time curve from time zero to the last quantifiable sampling time point $\left(T_{\text {last }}\right), A U C_{\infty}$ area under the plasma concentration-time curve from time zero to infinity, extrapolated from $T_{\text {last }}$ to infinity based on the terminal elimination rate constant $\lambda_{\mathrm{z}}, A e_{0-24}$ amount excreted in urine from time zero to $24 \mathrm{~h}$ post-dose, $C I$ confidence interval, $C L_{R(0-24)}$ renal clearance from time zero to $24 \mathrm{~h}$ post-dose

atorvastatin pharmacokinetics following lesinurad single dosing suggest there was no clinically relevant inhibition of OATP1B1 activity by lesinurad in vivo. It should be noted that these results should also be applicable to chronic, once-daily treatment with lesinurad, since lesinurad does not accumulate during multiple once-daily dosing. The results with lesinurad are in contrast to those with rifampin (rifampicin), a strong inhibitor of OATP1B1 in which an intravenous infusion of $600 \mathrm{mg}$ of rifampin $30 \mathrm{~min}$ before a single atorvastatin $40 \mathrm{mg}$ oral dose increased the exposure of atorvastatin by 3.9- to 6.8-fold [16]. The difference is not readily explained by differences in in vitro $\mathrm{IC}_{50}$ values (lesinurad, $\sim 9 \mu \mathrm{M}$; rifampin, $\sim 3 \mu \mathrm{M}$ ) or maximum plasma drug concentration (lesinurad, $\sim 44 \mathrm{nM}$; rifampin, $\sim 17 \mathrm{nM})$. However, rifampin has lower protein binding $(80 \%)$ than lesinurad $(98 \%)$ so the free concentration available to inhibit OATP is higher for rifampin than for lesinurad. In addition, different substrates were used to determine the $\mathrm{IC}_{50}$ values, estradiol-glucuronide for lesinurad and atorvastatin for rifampin. OATPs have been shown to have multiple binding sites [18]. Rifampin and atorvastatin compete for the same binding site whereas lesinurad and atorvastatin do not, and this difference may be responsible for the difference between lesinurad and rifampin on atorvastatin pharmacokinetics in vivo. Since atorvastatin was not used to test the inhibitory potential of lesinurad in vitro, the in vitro did not predict well the lack of clinical DDI between lesinurad and atorvastatin.

The plasma exposure of metformin $850 \mathrm{mg}$ was unchanged in the presence of lesinurad. The transporters that govern metformin pharmacokinetics and pharmacodynamics are different [19]. The elimination of metformin is solely via renal secretion (OCT2 and MATEs). Since lesinurad does not inhibit the kidney transporters involved in metformin renal clearance, the lack of metformin pharmacokinetics modulation by lesinurad was not unexpected. These recent data [19], which were not available at the time this study was designed, suggest that OCT1 interactions with metformin can only be detected by monitoring its pharmacodynamic effect. The distribution of metformin into the liver is mainly a pharmacodynamic effect as liver is the target organ for efficacy and metformin does not undergo extensive metabolism or biliary excretion. The liver transporter OCT1 was identified as potentially being inhibited by lesinurad, which could affect the pharmacodynamics of metformin. However, determination of 
Table 4 Analyses of urinary parameters of furosemide following administration of a single dose of furosemide in the absence or presence of lesinurad $400 \mathrm{mg}(n=11)$

\begin{tabular}{|c|c|c|c|c|c|}
\hline \multirow[t]{2}{*}{ Urinary parameter } & \multirow[t]{2}{*}{ Time point $(\mathrm{h})$} & \multicolumn{2}{|l|}{ LS mean } & \multirow{2}{*}{$\begin{array}{l}\text { Difference of LS } \\
\text { means }(95 \% \mathrm{CI})\end{array}$} & \multirow[t]{2}{*}{$p$ value } \\
\hline & & $\begin{array}{l}\text { Furosemide } 40 \mathrm{mg}+ \\
400 \mathrm{mg} \text { lesinurad }\end{array}$ & $\begin{array}{l}\text { Furosemide } \\
40 \mathrm{mg}\end{array}$ & & \\
\hline \multirow[t]{3}{*}{ Urine flow rate $(\mathrm{mL} / \mathrm{min})$} & $0-6$ & 6.64 & 6.95 & $-0.31(-1.73,1.11)$ & 0.6541 \\
\hline & $6-12$ & 2.23 & 2.58 & $-0.35(-1.47,0.78)$ & 0.5273 \\
\hline & $12-24$ & 1.36 & 1.17 & $0.19(-0.18,0.57)$ & 0.2999 \\
\hline \multirow[t]{3}{*}{ Urine volume $(\mathrm{mL})$} & $0-6$ & 2390.91 & 2502.27 & $-111.36(-622.06,399.34)$ & 0.6541 \\
\hline & $6-12$ & 804.55 & 929.55 & $-125.00(-530.27,280.27)$ & 0.5273 \\
\hline & $12-24$ & 981.82 & 843.18 & $138.64(-133.08,410.36)$ & 0.2999 \\
\hline \multirow[t]{3}{*}{ Urine sodium $(\mathrm{mmol} / \mathrm{L})$} & $0-6$ & 62.00 & 62.36 & $-0.36(-10.83,10.10)$ & 0.9429 \\
\hline & $6-12$ & 37.27 & 32.09 & $5.18(-6.50,16.87)$ & 0.3659 \\
\hline & $12-24$ & 26.45 & 30.55 & $-4.09(-14.13,5.95)$ & 0.4053 \\
\hline \multirow[t]{3}{*}{ Urine specific gravity } & $0-6$ & 0.99 & 0.99 & $0.00(-0.01,0.01)$ & 0.7348 \\
\hline & $6-12$ & 0.99 & 0.98 & $0.01(-0.01,0.03)$ & 0.1912 \\
\hline & $12-24$ & 0.98 & 0.99 & $-0.01(-0.03,0.01)$ & 0.4303 \\
\hline
\end{tabular}

$C I$ confidence interval, $h$ hours, $L S$ least squares

metformin pharmacodynamics was not considered at the time of study design.

Lesinurad reduced furosemide exposure as reflected by the decrease in furosemide $C_{\max }$ and $\mathrm{AUC}$ and the decreased urinary excretion of furosemide. However, the $\mathrm{CL}_{\mathrm{R}}$ of furosemide was unaffected by co-administration of lesinurad, denoted by the fact that $90 \%$ CIs of the GMR for $\mathrm{CL}_{\mathrm{R}}$ remained within the $80-125 \%$ boundaries. Thus, the reduced exposure is likely due to interference with furosemide absorption by lesinurad. Despite the reduced exposure, the diuretic activity of furosemide was unaffected. A similar lack of effect on the diuretic activity of furosemide, despite reduced furosemide exposure, has been reported with co-administration of the angiotensin II antagonist valsartan [20], a known inhibitor of OAT1/3 [10]. Overall, the results with furosemide do not support an effect of lesinurad on OAT1 or OAT3 activity.

\section{Conclusion}

In vitro studies suggested a potential interaction of lesinurad with the transporters OATP1B1, OCT1, and OAT1/3. However, in healthy subjects, no clinically relevant DDIs were found between lesinurad and atorvastatin, metformin, or furosemide, drugs that are known substrates for the respective transporters. Therefore, no DDIs are anticipated in patients with gout who are taking lesinurad and these concomitant drugs.

Acknowledgments Funding was provided by Ardea Biosciences/ AstraZeneca. Editorial support was provided by Dr. Anusha Bolonna and Tom Claus, PhD, of PAREXEL and funded by AstraZeneca.

\section{Compliance with Ethical Standards}

Conflict of interest Z. Shen and B. Kerr are employees of Ardea Biosciences, Inc., a member of the AstraZeneca Group. L.-T. Yeh, K. Wallach, and N. Zhu are former employees of Ardea Biosciences, Inc. M. Gillen is an employee of AstraZeneca.

Ethical approval The studies were conducted in accordance with the Declaration of Helsinki and International Conference on Harmonisation Good Clinical Practice (ICH GCP) guidelines.

Informed consent The protocol, protocol amendments, and written informed consent form were approved by an institutional review board. Subjects provided written informed consent prior to study participation.

Open Access This article is distributed under the terms of the Creative Commons Attribution-NonCommercial 4.0 International License (http://creativecommons.org/licenses/by-nc/4.0/), which permits any noncommercial use, distribution, and reproduction in any medium, provided you give appropriate credit to the original author(s) and the source, provide a link to the Creative Commons license, and indicate if changes were made.

\section{References}

1. Perez-Ruiz F. Treating to target: a strategy to cure gout. Rheumatology (Oxford). 2009:48(Suppl 2):ii9-ii14.

2. Khanna D, Fitzgerald JD, Khanna PP, Bae S, Singh MK, Neogi T, et al. 2012 American College of Rheumatology guidelines for management of gout. Part 1: systematic nonpharmacologic and pharmacologic therapeutic approaches to hyperuricemia. Arthritis Care Res (Hoboken). 2012;64(10):1431-46.

3. Richette P, Doherty M, Pascual E, Barskova V, Becce F, Coyfish $\mathrm{M}$, et al. Updated EULAR evidence-based recommendations for the management of gout. Ann Rheum Dis. 2014;73(Suppl 2):783 (Abstract SAT0531).

4. Zhang W, Doherty M, Bardin T, Pascual E, Barskova V, Conaghan $\mathrm{P}$, et al. EULAR evidence based recommendations for 
gout. Part II: Management. Report of a task force of the EULAR Standing Committee for International Clinical Studies Including Therapeutics (ESCISIT). Ann Rheum Dis. 2006;65(10):1312-24.

5. Yeh L, Shen Z, Kerr B, Tamai I, Hingorari V, Ong V, et al. RDEA594: a potent URAT1 inhibitor without affecting other important renal transporters, OAT1 and OAT3. Ann Rheum Dis. 2009;68(Suppl 3):320 (Abstract THU0452).

6. Fleischmann R, Kerr B, Yeh LT, Suster M, Shen Z, Polvent E, et al. Pharmacodynamic, pharmacokinetic and tolerability evaluation of concomitant administration of lesinurad and febuxostat in gout patients with hyperuricaemia. Rheumatology (Oxford). 2014;53(12):2167-74.

7. Kusuhara H, Sugiyama Y. In vitro-in vivo extrapolation of transporter-mediated clearance in the liver and kidney. Drug Metab Pharmacokinet. 2009;24(1):37-52.

8. Kalliokoski A, Niemi M. Impact of OATP transporters on pharmacokinetics. Br J Pharmacol. 2009;158(3):693-705.

9. US Department of Health and Human Services Food and Drug Administration Center for Drug Evaluation and Research (CDER). Drug interaction studies-study design, data analysis, implications for dosing, and labeling recommendations. Draft guidance. FDA Web site. Available from: http://www.fda.gov/ downloads/drugs/guidancecomplianceregulatoryinformation/guidances/ucm292362.pdf. Accessed 14 Sept 2015.

10. Sato M, Iwanaga T, Mamada H, Ogihara T, Yabuuchi H, Maeda $\mathrm{T}$, et al. Involvement of uric acid transporters in alteration of serum uric acid level by angiotensin II receptor blockers. Pharm Res. 2008;25(3):639-46.

11. Zhang Y, Warren MS, Zhang X, Diamond S, Williams B, Punwani $\mathrm{N}$, et al. Impact on creatinine renal clearance by the interplay of multiple renal transporters: a case study with INCB039110. Drug Metab Dispos. 2015;43(4):485-9.

12. Khosravan R, Wu JT, Joseph-Ridge N, Vernillet L. Pharmacokinetic interactions of concomitant administration of febuxostat and NSAIDs. J Clin Pharmacol. 2006;46(8):855-66.
13. Terkeltaub RA, Furst DE, Digiacinto JL, Kook KA, Davis MW. Novel evidence-based colchicine dose-reduction algorithm to predict and prevent colchicine toxicity in the presence of cytochrome P450 3A4/P-glycoprotein inhibitors. Arthritis Rheum. 2011;63(8):2226-37.

14. Lepist EI, Zhang X, Hao J, Huang J, Kosaka A, Birkus G, et al. Contribution of the organic anion transporter OAT2 to the renal active tubular secretion of creatinine and mechanism for serum creatinine elevations caused by cobicistat. Kidney Int. 2014;86 (2):350-7.

15. Motohashi $\mathrm{H}$, Inui $\mathrm{K}$. Multidrug and toxin extrusion family SLC47: physiological, pharmacokinetic and toxicokinetic importance of MATE1 and MATE2-K. Mol Aspects Med. 2013;34(2-3):661-8.

16. Lau YY, Huang Y, Frassetto L, Benet LZ. Effect of OATP1B transporter inhibition on the pharmacokinetics of atorvastatin in healthy volunteers. Clin Pharmacol Ther. 2007;81(2):194-204.

17. LIPITOR [prescribing information]. New York, NY: Pfizer Inc., 2009. Available at: http://labeling.pfizer.com/ShowLabeling. aspx $?$ id $=587$.

18. Noe J, Portmann R, Brun ME, Funk C. Substrate-dependent drug-drug interactions between gemfibrozil, fluvastatin and other organic anion-transporting peptide (OATP) substrates on OATP1B1, OATP2B1, and OATP1B3. Drug Metab Dispos. 2007;35(8):1308-14.

19. Zamek-Gliszczynski MJ, Bao JQ, Day JS, Higgins JW. Metformin sinusoidal efflux from the liver is consistent with negligible biliary excretion and absence of enterohepatic cycling. Drug Metab Dispos. 2013;41(11):1967-71.

20. Bindschedler M, Degen P, Flesch G, de GM, Preiswerk G. Pharmacokinetic and pharmacodynamic interaction of single oral doses of valsartan and furosemide. Eur J Clin Pharmacol. 1997;52(5):371-8. 